\title{
PROGRAMMABLE AUTOMATED WELDING SYSTEM (PAWS)
}

\section{7 \\ $p^{6}$}

\author{
Martin D. Kline \\ Project Manager \\ Babcock \& Wilcox, CIM Systems
}

Lynchburg, VA

N94- 30570

\begin{abstract}
An ambitious project to develop an advanced, automated welding system is being funded as part of the Navy Joining Center with Babcock \& Wilcox as the prime integrator. This program, the Programmable Automated Welding System (PAWS), involves the integration of both planning and real-time control activities. Planning functions include the development of a graphical decision support system within a standard, portable environment. Real-time control functions include the development of a modular, intelligent, real-time control system and the integration of a number of welding process sensors.
\end{abstract}

This paper presents each of these components of the PAWS and discusses how they can be utilized to automate the welding operation.

\section{Introduction}

The demands of small batch operations and the need to integrate into a wider automation strategy have pushed the development of advanced robotic and process control systems. One such system, presently directed specifically at welding applications, is under development by Babcock \& Wilcox. This approach addresses integrating both off-line planning and real-time control activities. This system was initially developed as an Advanced Technology Development program with the Naval Surface Warfare Center, Carderock Division, and is presently in an industrial transition phase as part of a Navy ManTech contract. This ManTech program is coordinated through the Navy Joining Center in Columbus, Ohio.

This advanced control system, known as the Programmable Automated Welding System (PAWS), has been created specifically to provide an automated means of planning, controlling, and evaluating critical welding situations to improve productivity and quality. The primary issue has been the desire to increase the cost-effectiveness and applicability of automation to difficult welding situations.

\section{System Overview}

PAWS consists of an Off-line Programming System (OLP) and an on-line, real-time controller. The OLP system provides a means to develop the plan for an entire automated welding operation, as well as the capability to manage existing plans. The OLP system provides an integrated platform for the motion and process planning functions. The Controller is capable of then implementing these plans during the actual welding process.

\section{PAWS Off-line Programming System}

The PAWS-OLPS resides on a UNIX-based workstation and is comprised of a relational database, a motion planning module, a geometric modeling system, and a job builder module. This system was developed following a client-server philosophy specifically to provide a decision support tool for the development, storage, and management of programs for the PAWS controller. The use of standards and the requirements of hardware portability have been highly stressed.

The PAWS-OLPS provides a means to develop and plan an entire automated robotic welding operation based on a computer aided design (CAD) model of the part to be welded. This planning occurs away from the robot and allows the robot system to maintain production while new applications are being planned and verified. 


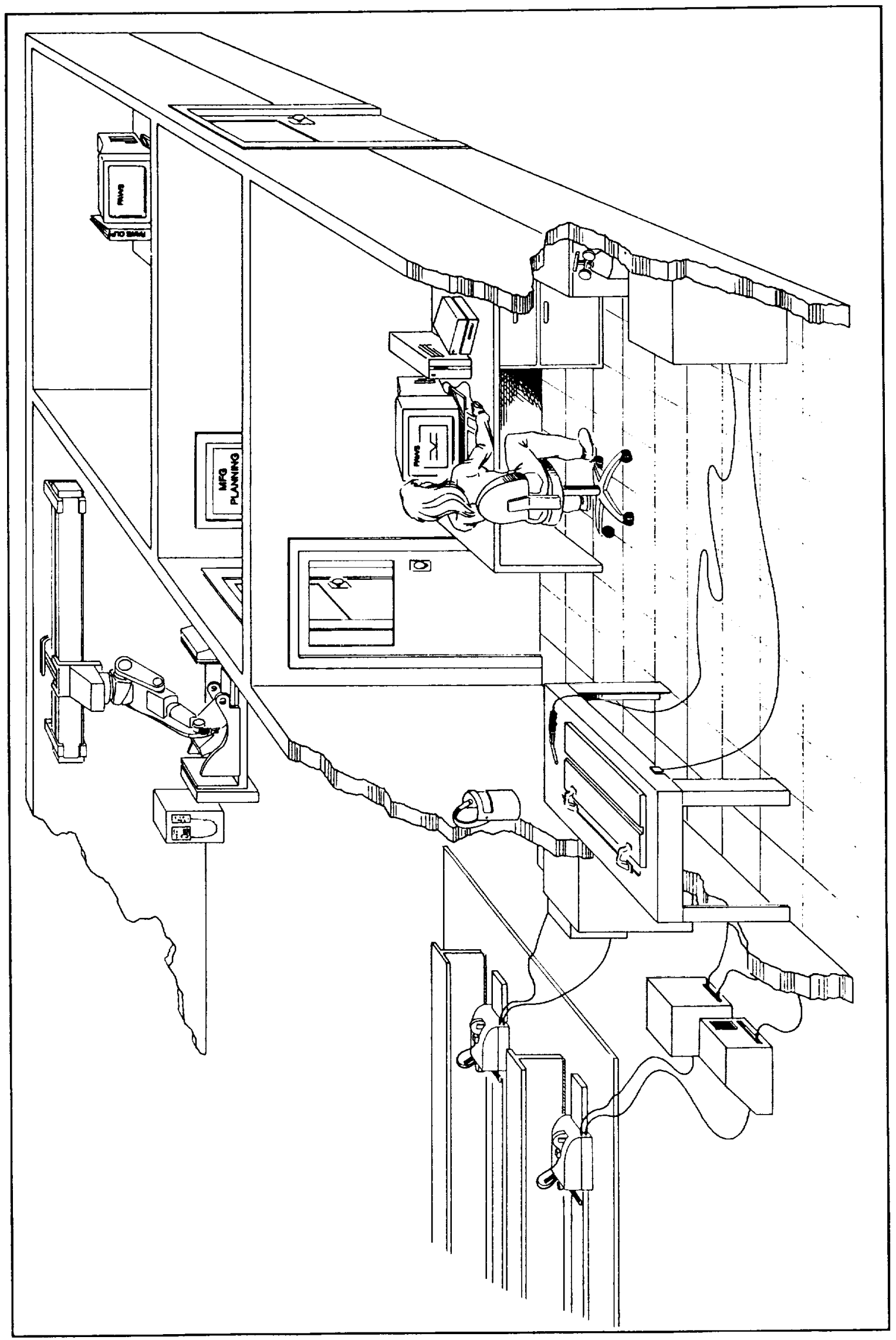

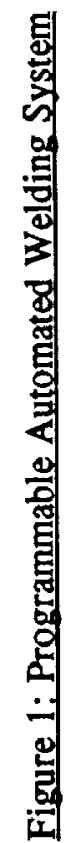


One OLPS can support multiple cells. OLPS is a key technology in small batch robotic operations. Without an OLPS, the economical utilization of robotic systems in small batch operations is not possible due to the nonproductive time required for the iterative nature of manual teaching methods.

The PAWS-OLPS provides a highly integrated approach to the planning of the welding and sensor operations. The welding application data is maintained in a relational database that is tightly coupled to the operation of the OLPS and the development of the overall plan.

Storage of certified welding procedures in a standard database format allows for the maintenance and re-use of previously performed welding trails. The client-server architecture allows the welding information to be maintained at a distributed computer by a knowledgeable resource. During subsequent planning operations, the OLPS presents to the planner only welding selections which are appropriate for the application at hand. By encapsulating and abstracting the welding knowledge, the PAWS-OLPS practically eliminates the need for the OLPS planner to be knowledgeable of the details of the welding process.

The OLPS also incorporates advanced motion planning and optimization methods. Functions are provided for optimal placement of the robot with respect to the workpiece and for automated determination of a collision-free path. Both the placement optimization and the collision avoidance capabilities are technologies critical to supporting small batch operations where accessibility limitations exist. The motion simulation ties the motion of the manipulator to the process information. This module provides a graphical 3-D animation of the manipulator performing the welding operation with real-time collision detection. A geometric modeler provides a convenient method for the modeling of parts, manipulators, end effectors, and physical environment constraints. The OLPS also provides a means of importing CAD files of components and generating solid models from those files. Once the plan is determined to be satisfactory, the plan is converted into a job to provide true off-line programming of the entire welding operation. This job is provided to the PAWS controller in the form of text files which are then converted to the controller's real-time database format.

Figure 1 depicts a setup in which the OLPS is being utilized to plan the operations for multiple devices. This concept envisions two track devices performing simple linear welds and an inverted robot arm coordinated with the motion of a 2-axis positioner. The welding engineer is developing and documenting procedures on a computer based in the weld lab. This interface enables the sharing of historical information and provides a dynamic means of managing weld procedures. Motion planning and simulation is then performed by the manufacturing planning department. The welding knowledge is referenced during this process.

\section{PAWS Controller}

The PAWS real-time robotic controller represents a state-of-the-art system based on a VME multi-processor platform. On-bus resources provide the interface to the process equipment via industrial $\mathrm{I} / \mathrm{O}$ (digital and analog), system I/O (serial and network interfaces, hard disk, monitor, etc..), and servo motion boards. This environmentally hardened platform supports the control of any common robotic manipulator and any arc welding process. In addition, the system has been designed to simultaneously accept input from a host of real-time sensors.

The key element of the controller is its flexibility. The controller and supporting peripheral components can be scaled to the technical needs of the application. This feature is supported both in software and by the ability to add processing power as dictated by the application. For example, the controller can be employed to control a simple 3-axis track mechanism or an articulated arm robot coordinated with a positioner. In addition, the controller can be configured to control a number of arc welding processes and sensors. In fact, given the proper welding equipment, multiple 
processes can even be maintained by a single controller. This flexibility enables the system to be extensible to emerging technologies such as new manipulators, welding processes and sensors.

The PAWS controller uses a real time database structure to compartmentalize the process data.
The controller is comprised of both kernel and expansion modules (refer to Figure 2). The kernel modules provide the base technologies for robotic process control. The expansion modules can then be selectively employed to address the specific process needs (e.g. welding).

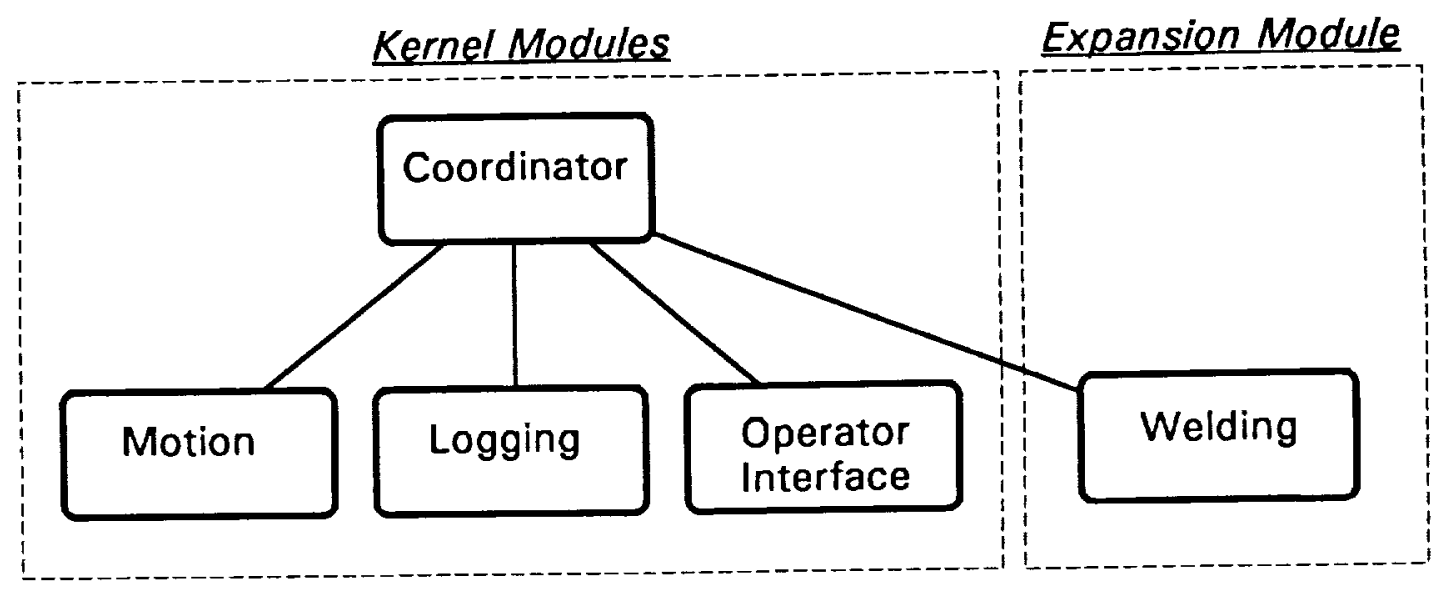

Figure 2: PAWS Controller Modules

\section{Kernel Modules}

Coordinator The coordinator module utilizes a script language to indicate the sequence of operation, and a rule-based expert system for exception handling. The sequence is built-up (either manually on the controller or automatically by the OLP's job builder module) as a series of statements specific to the process. These statements are English-like commands specific to the process at hand (e.g. START WELD, STOP WELD, MOVE ALONG, LOG DATA, etc..). This provides a readable, highlevel view of the job plan. During execution, the exception handler monitors the state of the ongoing process and issues programmed responses when anomalous conditions occur. These responses can range from simple warnings to complex adaptive responses.

Operator Interface All setup, walk-through teaching, and execution interaction with the operator is performed through an industrial pendant. This pendant consists of a portable, hand-held device with both push-buttons and a high-resolution graphical display. In appropriate situations, the pendant provides the operator the capability to adjust the process parameters during execution. To supplement the OLPS, an on-line editing capability is provided. The editor employs a portable PC and provides the ability to both edit and create jobs. In addition, this PC can be used to chart and analyze all logged data.

Motion The PAWS controller is capable of controlling a variety of manipulators, from a simple track device up to multi-axis robotic manipulators. A total of three manipulators and 32-axis may be controlled from a single controller. The 4 year ManTech program intends to develop and implement a production system in which a single controller is coordinating the operations of two or three robots simultaneously. Successful demonstration of this capability will provide a substantial cost advantage by sharing both hardware and manpower. A single operator can then be leveraged to monitor several operations at once. 
The motion module also incorporates the ability to perform path memorization: to retrace, with or without an offset bias, a modified path.

Additional features include: seam tracking, the ability to accept operator overrides of both Tool $Y$ (cross seam) and Tool $Z$ (standoff) distances, and the ability to modify motion parameters for adaptive control.

Logging The logging module allows for the selective logging of data based upon time, distance, or the reaching of an established threshold (e.g. heat input). Numeric data can be also be averaged while being logged.

\section{Expansion Module}

Welding The welding module commands the power supply to control the weld process. Currently, the system has been established to control the gas metal arc process (GMAW). The module commands and monitors a number of process parameters (e.g. current, voltage, wire feed speed, etc.). Process parameters are prevented from exceeding the limits established in the weld procedure. Additional general features include, consumable tracking and monitoring, user-definable $\mathrm{I} / \mathrm{O}$, and the expandability to other processes.
Adaptive parameter modifications are determined based upon input from sensors and from the operator. These may be direct parameter offsets (e.g. lower current 5 amps) or they may be in the form of indirect adjustments (e.g. increase bead width by $10 \%$ ). Indirect adjustments are processed by the internal process model into the appropriate parameter offsets. This model also resolves conflicts between adjustment sources. This resolution is performed by evaluating both priorities and constraints. One elegant feature of this implementation is the ability for the operator to adjust the indirect parameters without knowledge of the necessary direct parameters. In other words, the operator can concentrate on physical characteristics, such as bead width, without needing to mentally calculate the necessary adjustments to current, voltage, and travel speed.

This module also handles all sensor interface issues. These include device-specific communications, user-programmable data filtering, and adaptive control of the motion module. Exception handling is performed by an embedded rules engine. The sensors currently being employed are listed in Table 1.

Table 1: Sensors

\begin{tabular}{|l|l|}
\hline \multicolumn{1}{|c|}{ SENSOR } & \multicolumn{1}{c|}{ USAGE } \\
\hline Joint Vision Sensor & $\begin{array}{l}\text { Seam Tracking } \\
\text { Joint Volume } \\
\text { Joint Shape }\end{array}$ \\
\hline Post Weld Geometry Sensor & $\begin{array}{l}\text { Pool Size } \\
\text { Pool Location }\end{array}$ \\
\hline Arc Element Sensor & Contamination in Arc $\left(\mathrm{H}_{2}, \mathrm{O}_{2}, \mathrm{Fe}\right)$ \\
\hline Through-the-Arc Sensing & Seam Tracking \\
\hline Touch Sensing & Joint Location \\
\hline
\end{tabular}


The listed sensors cover a wide range of control areas including feed forward, feedback, and process monitoring. The PAWS controller is capable, however, of being configured to utilize only those sensors which are needed to perform the particular application. A typical application which is severely space-limited may use only through-the-arc sensing, whereas, an accessible component with critical process control criteria may utilize three or four different sensors.

\section{Summary}

The 30 month-long ATD phase of the PAWS program ended in November 1992 and the follow-on 4 year ManTech program was started in September 1993. The system will be industrially hardened during the first year of this program and will be applied in an Navy Joining Center Teaching Factory at B\&W CIM Systems in Lynchburg, VA. The technology will be implemented into production systems during 1995. Follow-on years will focus upon expansion of the technology based upon end-user needs. This will include expansion into other welding processes (e.g. FCAW, GTAW, PAW), the support of multiple robots, expanded exception handling techniques, and the integration of design data directly into the OLP. In addition, the architecture is being developed for application to other non-welding robotic processes (e.g. inspection, surface finishing, cleaning). 